\title{
Estimativas de adaptabilidade e estabilidade fenotípicas em características produtivas em bovinos de corte mestiços criados em diferentes ambientes
}

[Phenotypic adaptability and stability in productive traits for crossbred beef cattle raised in different environments]

\author{
J.B.G. Barros ${ }^{1}$, J.C.C. Balieiro ${ }^{2 *}$, J.P. Eler ${ }^{2}$, J.B.S. Ferraz ${ }^{2}$, E.S. Balieiro ${ }^{3}$, E.C. Mattos $^{2}$ \\ ${ }^{1}$ Médico veterinário autônomo \\ ${ }^{2}$ Faculdade de Zootecnia e Engenharia de Alimentos - USP \\ Av. Duque de Caxias Norte, 225 \\ Caixa Postal 23 \\ 13635-970 - Pirassununga, SP \\ ${ }^{3}$ Instituto de Zootecnia - UFRRJ - Seropédica, RJ.
}

\begin{abstract}
RESUMO
Foram analisados 39.212 registros para peso à desmama e 16.546 registros para peso aos 12 meses e ganho de peso até 160 dias pós-desmama, com a finalidade de avaliar a utilização de metodologia para discriminar estimativas de parâmetros de adaptabilidade e estabilidade fenotípicas. Nove composições genotípicas foram distribuídas em seis ambientes para peso à desmama. Para peso aos 12 meses e ganho de peso até 160 dias pós-desmama, utilizaram-se cinco ambientes. As estimativas de médias, desviospadrão e coeficientes de variação para peso à desmama, peso aos 12 meses e ganho de peso até 160 dias pós-desmama foram: $204,3 \pm 22,6 \mathrm{~kg}$ e $11,1 \% ; 280,4 \pm 25,9 \mathrm{~kg}$ e $9,2 \%$ e $164,8 \pm 18,1 \mathrm{~kg}$ e $28,0 \%$, respectivamente. Verificou-se dependência entre principais combinações genotípicas e ambientes avaliados, o que impediu a recomendação geral das melhores combinações genotípicas para todos os ambientes. As análises de adaptabilidade mostraram ser eficientes em discriminar o comportamento das combinações genotípicas dentro de cada ambiente avaliado.
\end{abstract}

Palavras-chave: gado de corte, genótipo x ambiente, adaptabilidade, estabilidade fenotípica

\begin{abstract}
Data from 39,212 weaning weights and 16,546 yearling weights and weight gain from weaning to 160 days after weaning, were used to evaluate a methodology to discriminate estimates of phenotypic adaptability and stability. Nine genotypic compositions distributed in six environments for weaning weight, and in five environments for yearling weight and weight gain from weaning to 160 days post weaning, were analyzed. The averages, standard deviations and coefficients of variation for weaning weight, yearling weight and weight gain from weaning to 160 days post weaning were: $204.3 \pm 22.6 \mathrm{~kg}$ and $11.1 \% ; 280.4 \pm 25.9 \mathrm{~kg}$ and $9.2 \%$; and $164.8 \pm 18.1 \mathrm{~kg}$ and $28 \%$, respectively. The genotype by environment interaction suggests that the best genotype combinations can not be recommended for all the environments. The adaptability analysis was efficient to discriminate genotype combination within environment.
\end{abstract}

Keywords: beef cattle, genotype x environmental, adaptability, stability

Recebido em 17 de março de 2004

Aceito em 28 de março de 2006

*Autor para correspondência (corresponding author)

E-mail: balieiro@usp.br 


\section{INTRODUÇÃo}

A manifestação fenotípica para determinada característica é o resultado da ação do genótipo e do meio ambiente. Entretanto, quando se consideram vários ambientes, pode-se detectar um componente adicional, causado pela interação genótipo x ambiente. Sua avaliação é de grande importância no melhoramento genético, pois ela pode indicar que o melhor genótipo em um ambiente não o ser no outro (Cruz e Regazzi, 1994; Cruz e Regazzi, 2001). Esse fato torna difícil recomendar combinações genotípicas favoráveis nas diferentes regiões do país.

Os estudos que trataram dos aspectos relacionados com a interação genótipo $\mathrm{x}$ ambiente utilizaram o resultado da interação touro $\mathrm{x}$ rebanho para expressar a magnitude do problema. Meyer (1987), que trabalhou com bovinos leiteiros, e Notter et al. (1992), com gado de corte, verificaram o comportamento da interação para a característica. A interação touro $\mathrm{x}$ região geográfica também foi estudada por Tess et al. (1979), ao trabalharem com características de desenvolvimento ponderal de bovinos para corte da raça Simental. Rorato et al. (1994) e Rorato et al. (1996) verificaram o impacto da interação touro $\mathrm{x}$ região na raça Holandesa, dentro do Estado do Paraná, e em diferentes regiões do Brasil, respectivamente.

Alterações nos componentes de co(variância) e, conseqüentemente, nos parâmetros genéticos, têm sido utilizadas como forma de avaliar a interação genótipo $\mathrm{x}$ ambiente. Ramos et al. (1996) e Torres et al. (2000) estudaram o problema na raça Holandesa no Brasil, assim como Ojango e Pollott (2002) e Zwald et al. (2003), também com a raça Holandesa, avaliaram as alterações nos componentes de (co) variância em países de clima temperado e tropical. Em características de desenvolvimento ponderal para bovinos de corte no Brasil, Balieiro (2001) e Ferreira et al. (2001) identificaram alterações nos parâmetros genéticos atribuídos à interação genótipo $\mathrm{x}$ ambiente em animais da raça Nelore. Mattos et al. (2000) e Lee e Bertrand (2002) ao trabalharem com a raça Hereford, e Newman et al. (2002), ao mensurarem características de desempenho e carcaça em bovinos de diversas raças, identificaram alterações dos componentes de (co) variância e parâmetros genéticos atribuídos à interação. A despeito de sua importância, esses estudos não forneceram maiores informações sobre o comportamento de combinações genotípicas de bovinos para corte, oriundos de cruzamentos, frente aos diferentes ambientes.

Segundo Cruz e Regazzi (2001), estratégias que permitem identificar as combinações genotípicas de comportamento previsível e que respondem positivamente às variações ambientais favoráveis são denominadas, respectivamente, de análises de adaptabilidade e estabilidade. Recentemente, Rezende et al. (1999) e Rezende e Ferreira (2001) compararam métodos de estimação da estabilidade fenotípica em animais de diferentes percentagens de alelos (31/32, GC1; GC2, GC3, de GC4 a GC11 e PO) na raça Holandesa. Os animais foram separados em 14 ambientes distintos do Estado de Minas Gerais e os métodos de análises para estimação dos parâmetros de estabilidade foram o da regressão (Eberhart e Russel, 1966; Cruz et al., 1989), o do desvio do desempenho máximo (Lins e Binns, 1988) e o additive multiplicative models interaction (AMMI), proposto por Gauch Junior e Zobel (1988). Os melhores métodos, segundo Rezende e Ferreira (2001), foram o da regressão e do desvio do desempenho máximo.

São escassos os trabalhos que avaliaram a interação genótipo $\mathrm{x}$ ambiente em gado de corte. Os objetivos deste trabalho foram avaliar $\mathrm{o}$ comportamento de algumas variáveis produtivas para grupos de combinações genotípicas de bovinos obtidos por cruzamento, considerandose a interação genótipo $\mathrm{x}$ ambiente, e avaliar $\mathrm{o}$ uso de metodologia para discriminar estimativas de parâmetros de adaptabilidade e estabilidade fenotípicas para bovinos mestiços, criados em diferentes ambientes.

\section{MATERIAL E MÉTODOS}

Os dados referem-se às características de desenvolvimento ponderal: peso à desmama (PD), peso aos 12 meses (P12) e ganho de peso até 160 dias pós desmama (GP160), oriundos de animais obtidos por cruzamento industrial Europeu-Zebu e criados em diferentes regiões do país. As combinações genotípicas, indicadas como diferentes porcentagens de alelos que 
compõem os animais, são apresentadas na Tab. 1. Os ambientes 1, 2, 3, 4, 5 e 6 representaram os municípios de Pontes Gestal (SP), Dois Irmãos do Butriti (MS) Guaraci (SP), Ivinhema (MS), Ponta Porá (MS) e Cumaru do Norte (PA), respectivamente.

Tabela 1. Combinações genotípicas e respectivas porcentagens de alelos dos animais avaliados

\begin{tabular}{|c|c|}
\hline Composição genotípica & Porcentagens de alelos nas combinações genotípicas \\
\hline 1 & $75 \%$ Zebu x $00 \%$ Adaptado x 25\% Britânico x 00\% Continental \\
\hline 2 & $25 \%$ Zebu x $25 \%$ Adaptado x $25 \%$ Britânico x $25 \%$ Continental \\
\hline 3 & $25 \%$ Zebu x $25 \%$ Adaptado x 50\% Britânico x 00\% Continental \\
\hline 4 & $25 \%$ Zebu x $50 \%$ Adaptado x $00 \%$ Britânico x $25 \%$ Continental \\
\hline 5 & $25 \%$ Zebu x $50 \%$ Adaptado x $25 \%$ Britânico x $00 \%$ Continental \\
\hline 6 & $50 \%$ Zebu x $00 \%$ Adaptado x 00\% Britânico x 50\% Continental \\
\hline 7 & $50 \%$ Zebu x $00 \%$ Adaptado x 25\% Britânico x 25\% Continental \\
\hline 8 & $50 \%$ Zebu x $00 \%$ Adaptado x 50\% Britânico x $00 \%$ Continental \\
\hline 9 & $50 \%$ Zebu x $50 \%$ Adaptado x $00 \%$ Britânico x $00 \%$ Continental \\
\hline
\end{tabular}

Foram analisados 39.212 registros para PD e 16.546 registros para P12 e GP160. Nove composições genotípicas foram distribuídas em cada um dos seis ambientes para PD. Para P12 e GP160, utilizaram-se apenas cinco ambientes, em virtude da pouca representatividade amostral para as composições genotípicas 6 e 7 no ambiente 3 .

Previamente ao estudo da interação genótipo $\mathrm{x}$ ambiente, foram realizadas análises de variância para cada ambiente, visando a avaliar a existência de variabilidade genotípica entre as combinações dos diferentes cruzamentos, a precisão relativa das informações de cada ambiente, verificada por meio dos coeficientes de variação dentro dos ambientes, e a homogeneidade das variâncias residuais.

Posteriormente, foram realizadas análises de variância conjunta envolvendo o grupo de combinações genotípicas e os vários ambientes para cada uma das características estudadas. Para PD adotou-se um modelo que contemplou os efeitos de combinação genotípica, ambiente, interação combinação genotípica $\mathrm{x}$ ambiente, grupo de contemporâneos dentro de ambiente, além das covariáveis idade da vaca (linear e quadrático) e idade do animal à desmama (linear e quadrático). Para as características P12 e GP160, foram utilizados os mesmos modelos, apenas com adaptações particulares a cada característica na modelagem dos efeitos e covariáveis. Para P12, utilizaram-se os mesmos efeitos e covariáveis, com idade do animal corrigida apenas para efeito linear. Para GP160, adotaram-se os mesmos efeitos e apenas a covariável idade da vaca ao parto com efeito linear e quadrático.

A decomposição da soma de quadrados da interação genótipo $\mathrm{x}$ ambiente, para cada variável estudada, foi realizada pelo método da regressão proposto por Eberhart e Russel (1966) e Cruz et al. (1989). Essas análises visaram a estimar os parâmetros de adaptabilidade (capacidade das combinações genotípicas aproveitarem com vantagem o estímulo do ambiente) e estabilidade (capacidade das combinações genotípicas mostrarem comportamento previsível em função do estímulo do ambiente). Foram utilizados os procedimentos PROC GLM e PROC VARCOMP (User's..., 1985), nas análises de variância conjuntas. Para as análises de adaptabilidade e estabilidade foram desenvolvidas rotinas de cálculos utilizando-se como ferramenta o Microsoft Excel ${ }^{\circledR}$, no sistema operacional Microsoft Windows $98 S E^{\circledR}$. Essas rotinas tomaram por base os procedimentos indicados por Cruz (2001) e Cruz e Regazzi, (2001).

\section{RESULTADOS E DISCUSSÃO}

As estimativas de médias, desvios-padrão e coeficientes de variação, obtidas nas análises de variância conjuntas para PD, P12 e GP160, foram: $204,3 \pm 22,6 \mathrm{~kg}$ e $11,1 \% ; 280,4 \pm 25,9 \mathrm{~kg}$ e $9,2 \% ; 164,8 \pm 18,1 \mathrm{~kg}$ e $28 \%$, respectivamente. Brown et al. (1993), ao analisarem o PD de animais Angus, Brahman e os cruzamentos 
recíprocos dessas raças, submetidos a dois sistemas de pastagens, verificaram valores que oscilaram entre $199,7 \pm 2,5 \mathrm{~kg}$ para Angus puros, e $231 \pm 3,1 \mathrm{~kg}$ para Brahman $\mathrm{x}$ Angus. Burrow (2001), ao estudar animais com 25\% Hereford, $25 \%$ Shorthorn e 50\% Africander (AX) e animais com $25 \%$ Hereford, 25\% Brahman, 25\% Shorthorn e 25\% Africander (AXBX), observou valores inferiores aos deste trabalho para $\mathrm{PD}$ e $\mathrm{P} 12$, de $182,0 \pm 26,4 \mathrm{~kg}$ e $222,0 \pm 32,7 \mathrm{~kg}$, respectivamente. Newman et al. (2002), ao avaliarem o peso aos 400 dias de idade de progênies oriundas de cruzamentos de touros Angus, Hereford, Shorthorn, Belmont Red e Santa Gertrudis, acasaladas com fêmeas Brahman, obtiveram estimativas que variaram de $268,7 \pm 2,7 \mathrm{~kg}$ para progênies de cruzamentos Belmont Red x Brahman, a 313,2 $\pm 3,5 \mathrm{~kg}$, para progênies de cruzamentos Hereford x Brahman.
As demais progênies apresentaram valores intermediários.

As análises de variância conjuntas para as características PD, P12 e GP160, encontram-se nas Tab. 2, 3 e 4, respectivamente.

Em virtude dos resultados significativos para as interações genótipo $\mathrm{x}$ ambiente nas três características avaliadas, procedeu-se à decomposição de cada soma de quadrados relativa a esta fonte de variação, visando a obter estimativas dos parâmetros de adaptabilidade e estabilidade, segundo a metodologia proposta por Eberhart e Russel (1966) e Cruz et al. (1989). Essas estimativas e seus respectivos níveis de significância, encontram-se nas Tab. 5, 6 e 7, respectivamente.

Tabela 2. Resumo da análise de variância conjunta para a característica peso à desmama (PD), considerando a interação genótipo $\mathrm{x}$ ambiente

\begin{tabular}{lccc}
\hline Fonte de variação & GL & QM & F \\
\hline Composição genotípica (CG) & 8 & $28.038,68$ & $54,95^{* *}$ \\
Ambiente (A) & 5 & $71.928,77$ & $140,96^{* *}$ \\
Interação CG x A & 40 & $6.980,78$ & $13,68^{* *}$ \\
Grupo de contemporâneo /ambiente & 1.127 & $8.622,33$ & $16,90 * *$ \\
$\quad$ Idade da vaca ao parto (linear) & 1 & $489.273,40$ & $958,84^{* *}$ \\
$\quad$ Idade da vaca ao parto (quadrático) & 1 & $520.292,28$ & $1.019,63 * *$ \\
$\quad$ Idade do animal à pesagem (linear) & 1 & $164.601,11$ & $322,57 * *$ \\
$\quad$ Idade do animal à pesagem (quadrático) & 1 & $26.248,52$ & $51,44 * *$ \\
Resíduo & 38.027 & 510,27 & \\
\hline
\end{tabular}

Tabela 3. Resumo da análise de variância conjunta para a característica peso aos 12 meses (P12), considerando a interação genótipo $\mathrm{x}$ ambiente

\begin{tabular}{lccc}
\hline Fonte de variação & GL & QM & F \\
\hline Composição genotípica (CG) & 8 & $25.682,19$ & $38,16^{* *}$ \\
Ambiente (A) & 4 & $26.383,76$ & $39,21^{* *}$ \\
Interação CG x A & 32 & $2.059,63$ & $3,06 * *$ \\
Grupo de contemporâneo /ambiente & 973 & $20.483,23$ & $30,44^{* *}$ \\
$\quad$ Idade da Vaca ao parto (linear) & 1 & $65.686,67$ & $97,61^{* *}$ \\
$\quad$ Idade da Vaca ao parto (quadrático) & 1 & $82.381,74$ & $122,42 * *$ \\
$\quad$ Idade do Animal (linear) & 1 & $1.281 .270,90$ & $1.903,95 * *$ \\
Resíduo & 15.525 & 672,25 & \\
\hline
\end{tabular}

\footnotetext{
** $(\mathrm{P}<0,01)$
} 
Tabela 4. Resumo da análise de variância conjunta para a característica ganho de peso até 160 dias pósdesmama (GP160), considerando a interação genótipo x ambiente

\begin{tabular}{lccc}
\hline Fonte de variação & $\mathrm{GL}$ & $\mathrm{QM}$ & $\mathrm{F}$ \\
\hline Composição genotípica (CG) & 8 & $4.980,98$ & $15,12^{* *}$ \\
Ambiente (A) & 4 & $31.258,39$ & $94,91 * *$ \\
Interação CG x A & 32 & 890,43 & $2,70 * *$ \\
Grupo de contemporâneo /ambiente & 973 & $12.241,08$ & $37,17 * *$ \\
$\quad$ Idade da Vaca ao parto (linear) & 1 & $24.397,99$ & $74,08 * *$ \\
$\quad$ Idade da Vaca ao parto (quadrático) & 1 & $17.597,39$ & $53,43 * *$ \\
Resíduo & 15.526 & 329,36 & \\
\hline
\end{tabular}

$* *(\mathrm{P}<0,01)$

Tabela 5. Estimativas dos parâmetros de adaptabilidade $\left(\hat{\beta}_{0(i)}\right.$ e $\left.\hat{\beta}_{1(i)}\right)$ e estabilidade $\left(\hat{\sigma}_{d(i)}^{2}\right)$ para peso à desmama $(\mathrm{PD})$ em nove composições genotípicas de bovinos de corte avaliadas em seis ambientes

\begin{tabular}{lcccc}
\hline Genótipo & $\hat{\beta}_{0(i)}$ & $\hat{\beta}_{1(i)}$ & $\hat{\sigma}^{2} d(i)$ & $R_{(i)}^{2}(\%)$ \\
\hline 1 & 184,05 & $1,02^{\mathrm{ns}}$ & $171,51^{* *}$ & 72,62 \\
2 & 221,33 & $0,96^{\mathrm{ns}}$ & $175,42^{* *}$ & 69,50 \\
3 & 211,22 & $0,81^{++}$ & $60,19^{* *}$ & 82,29 \\
4 & 207,55 & $1,01^{\mathrm{ns}}$ & $104,06^{* *}$ & 81,03 \\
5 & 205,44 & $0,87^{++}$ & $86,73^{* *}$ & 79,03 \\
6 & 195,36 & $1,21^{++}$ & $118,01 * *$ & 84,33 \\
7 & 188,00 & $0,85^{++}$ & $222,60^{* *}$ & 58,71 \\
8 & 206,33 & $1,03^{\mathrm{ns}}$ & $11,11^{* *}$ & 97,52 \\
9 & 193,77 & $1,22^{++}$ & $33,04 * *$ & 95,06 \\
\hline
\end{tabular}

ns $(\mathrm{P}>0,05) ;{ }^{++}(\mathrm{P}<0,01)$, teste $t ; * *(\mathrm{P}<0,01)$ teste $\mathrm{F}$.

Tabela 6. Estimativas dos parâmetros de adaptabilidade $\left(\hat{\beta}_{0(i)}\right.$ e $\left.\hat{\beta}_{1(i)}\right)$ e estabilidade $\left(\hat{\sigma}_{d(i)}^{2}\right)$ para peso aos 12 meses (P12) em nove composições genotípicas de bovinos de corte avaliadas em cinco ambientes

\begin{tabular}{lcccc}
\hline Genótipo & $\hat{\beta}_{0(i)}$ & $\hat{\beta}_{1(i)}$ & $\hat{\sigma}^{2}{ }_{(i)}$ & $R_{(i)}^{2}(\%)$ \\
\hline 1 & 249,53 & $1,67^{++}$ & $271,60 * *$ & 35,59 \\
2 & 307,03 & $-0,96^{++}$ & $271,49 * *$ & 15,36 \\
3 & 295,33 & $0,53^{++}$ & $34,70^{* *}$ & 29,20 \\
4 & 282,99 & $1,23^{\mathrm{ns}}$ & $270,23 * *$ & 23,15 \\
5 & 294,10 & $-0,23^{++}$ & $271,09 * *$ & 1,06 \\
6 & 249,00 & $2,16^{++}$ & $203,08^{* *}$ & 55,17 \\
7 & 241,34 & $0,00^{++}$ & $635,79^{* *}$ & 0,00 \\
8 & 265,51 & $3,05^{++}$ & $150,08^{* *}$ & 76,88 \\
9 & 265,91 & $1,55^{++}$ & $147,77^{* *}$ & 46,51 \\
\hline ns $(\mathrm{P}>0,05) ;{ }^{++}(\mathrm{P}<0,01)$, teste $t ; * *(\mathrm{P}<0,01)$ teste $\mathrm{F}$. & &
\end{tabular}


Tabela 7. Estimativas dos parâmetros de adaptabilidade $\left(\hat{\beta}_{0(i)}\right.$ e $\left.\hat{\beta}_{1(i)}\right)$ e estabilidade $\left(\hat{\sigma}^{2} d(i)\right.$ ) para ganho de peso até 160 dias pós-desmama (GP160) em nove composições genotípicas de bovinos de corte avaliadas em cinco ambientes

\begin{tabular}{lcccc}
\hline Genótipo & $\hat{\beta}_{0(i)}$ & $\hat{\beta}_{1(i)}$ & $\hat{\sigma}^{2} d(i)$ & $R_{(i)}^{2}(\%)$ \\
\hline 1 & 61,79 & $1,39^{++}$ & $82,13^{* *}$ & 88,40 \\
2 & 73,25 & $1,02^{\mathrm{ns}}$ & $52,95^{* *}$ & 86,30 \\
3 & 72,94 & $1,26^{++}$ & $13,19^{* *}$ & 97,34 \\
4 & 65,67 & $1,25^{++}$ & $58,22^{* *}$ & 89,58 \\
5 & 72,66 & $1,28^{++}$ & $40,19^{* *}$ & 92,91 \\
6 & 58,96 & $0,77^{++}$ & $27,88^{* *}$ & 87,16 \\
7 & 55,33 & $0,59^{++}$ & $46,76^{* *}$ & 70,68 \\
8 & 58,93 & $0,60^{++}$ & $35,38^{* *}$ & 76,32 \\
9 & 57,71 & $0,83^{++}$ & $164,05^{* *}$ & 57,41 \\
\hline
\end{tabular}

ns $(\mathrm{P}>0,05) ;{ }^{++}(\mathrm{P}<0,01)$, teste $t ; * *(\mathrm{P}<0,01)$ teste $\mathrm{F}$.

Para PD, os interceptos $\left(\hat{\beta}_{0(i)}\right)$ representam os pesos médios à desmama nas diferentes composições genótípicas. Os genótipos 2, 3 e 4 resultaram nos maiores PD e os genótipos 1, 7 e 9 os de menores PD. Com base no teste $t$ de significância para os coeficientes angulares $\left(\hat{\beta}_{1(i)}\right)$, corrigidos para os índices ambientais de cada ambiente avaliado, verificou-se que os animais de composição genotípica 1, 2, 4 e 8 foram considerados como de adaptabilidade geral. Os de genótipos 6 e 9 foram considerados de adaptabilidade específica a ambientes favoráveis. As composições genotípicas 3, 5 e 7 foram classificadas como animais com adaptabilidade específica a ambientes desfavoráveis. O comportamento das composições genotípicas de acordo com cada índice ambiental, para PD, encontra-se na Fig.1.

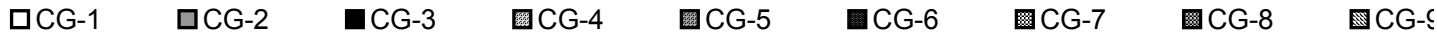

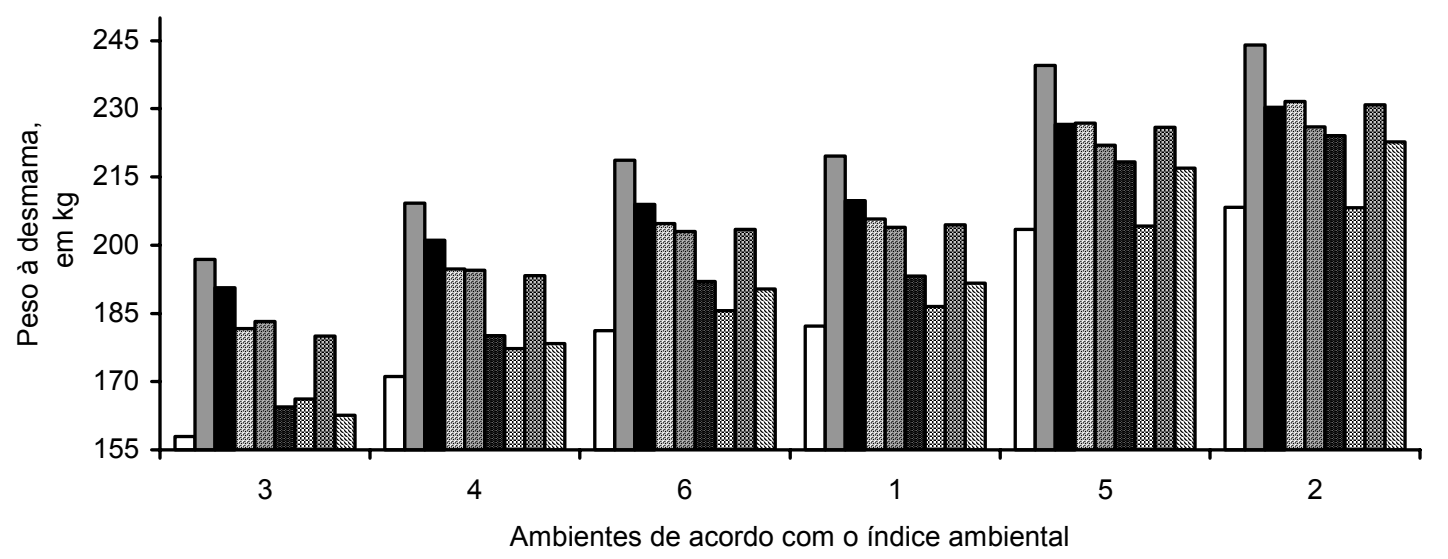

Figura 1. Comportamento da composição genotípica (CG-i) de acordo com cada índice ambiental, para a característica peso à desmama (PD) 
Os componentes de variância atribuídos aos desvios da regressão $\left(\hat{\sigma}_{d(i)}^{2}\right)$, para todas as combinações genotípicas sugeriram, que os genótipos seriam de estabilidade baixa. Nesses casos, uma medida auxiliar de comparação deve ser utilizada, sendo recomendado o coeficiente de determinação $\left(\mathrm{R}_{(\mathrm{i})}^{2}\right)$. Por esse critério, os genótipos 8 e 6 não deveriam ser julgados totalmente indesejáveis, pois apresentaram pesos à desmama medianos e seus respectivos $R_{(i)}^{2}$ atingiram níveis elevados.

O P12 evidenciou melhores interceptos $\left(\hat{\beta}_{0(i)}\right)$ para as combinações genotípicas 2,3 e 5, traduzindo-se como os de maiores pesos médios nessa característica, enquanto que os genótipos 7 , 6 e 1 foram os de menores P12. Os coeficientes angulares $\left(\hat{\beta}_{1(i)}\right)$ para essa característica indicaram que os únicos animais com adaptabilidade geral foram os de composição genotípica 4. Os animais de combinações genotípicas 1, 6, 8 e 9 foram classificados como de adaptabilidade específica a ambientes favoráveis. Os genótipos 2, 3, 5 e 7 o foram como animais com adaptabilidade específica a ambientes desfavoráveis. O comportamento das composições genotípicas, de acordo com cada índice ambiental, para P12, encontra-se na Fig. 2.
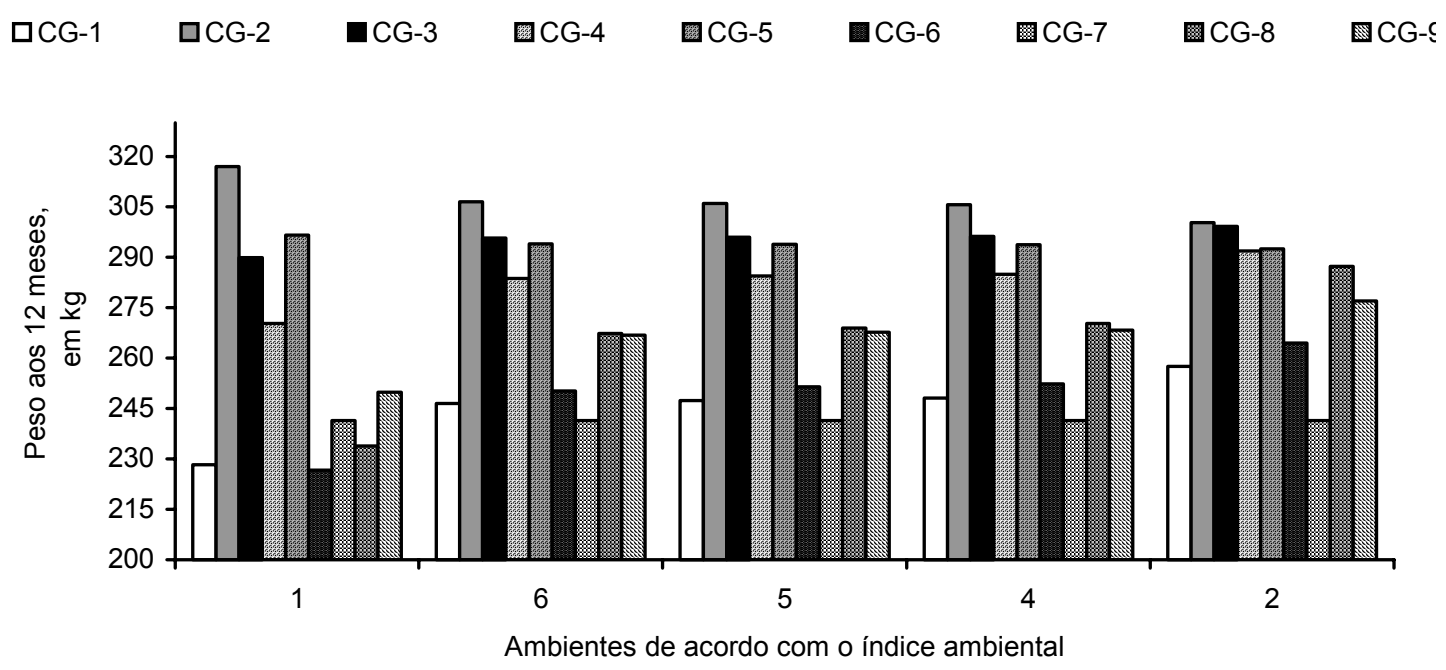

Figura 2. Comportamento da composição genotípica (CG-i) de acordo com cada índice ambiental, para a característica peso aos 12 meses (P12)

Os componentes de variância atribuídos aos desvios da regressão $\left(\hat{\sigma}_{d(i)}^{2}\right)$, para todas as combinações genotípicas avaliadas, também indicaram que os genótipos seriam de estabilidade baixa. Utilizando o critério dos $\mathrm{R}_{(\mathrm{i})}^{2}$, somente o genótipo 8 não deveria ser julgado totalmente indesejável, uma vez que apresentou P12 mediano e seu $\mathrm{R}_{(\mathrm{i})}^{2}$ foi considerado alto.

Para a característica GP160, os interceptos $\left(\hat{\beta}_{0(i)}\right)$ para as combinações genotípicas 2,3 e 5 representaram os de maior ganho, enquanto que os genótipos 7, 9, 8 e 6 foram os de menor ganho, respectivamente (Tab. 7). Os coeficientes angulares $\left(\hat{\beta}_{1(i)}\right)$ sugeriram que os únicos animais classificados como de adaptabilidade geral foram os de composição genotípica 2. Os de combinações genotípicas 1, 3, 4 e 5 foram considerados de adaptabilidade específica a ambientes favoráveis. Os de composição genotípica $6,7,8$ e 9 foram classificados de adaptabilidade específica a ambientes desfavoráveis. $\mathrm{O}$ comportamento das 
composições genotípicas, de acordo com cada índice ambiental para GP160, encontra-se na Fig. 3. Pelos componentes de variância atribuídos aos desvios da regressão $\left(\hat{\sigma}_{d(i)}^{2}\right)$, todas as combinações genotípicas avaliadas também foram classificadas como de estabilidade baixa. Utilizando o critério do $\mathrm{R}_{(\mathrm{i})}^{2}$, os genótipos 3 e 5 poderiam ser recomendados por apresentarem maior GP160, além de $\mathrm{R}_{(\mathrm{i})}^{2}$ considerado elevado.

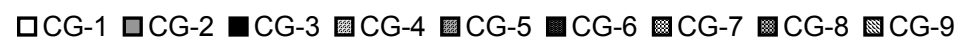

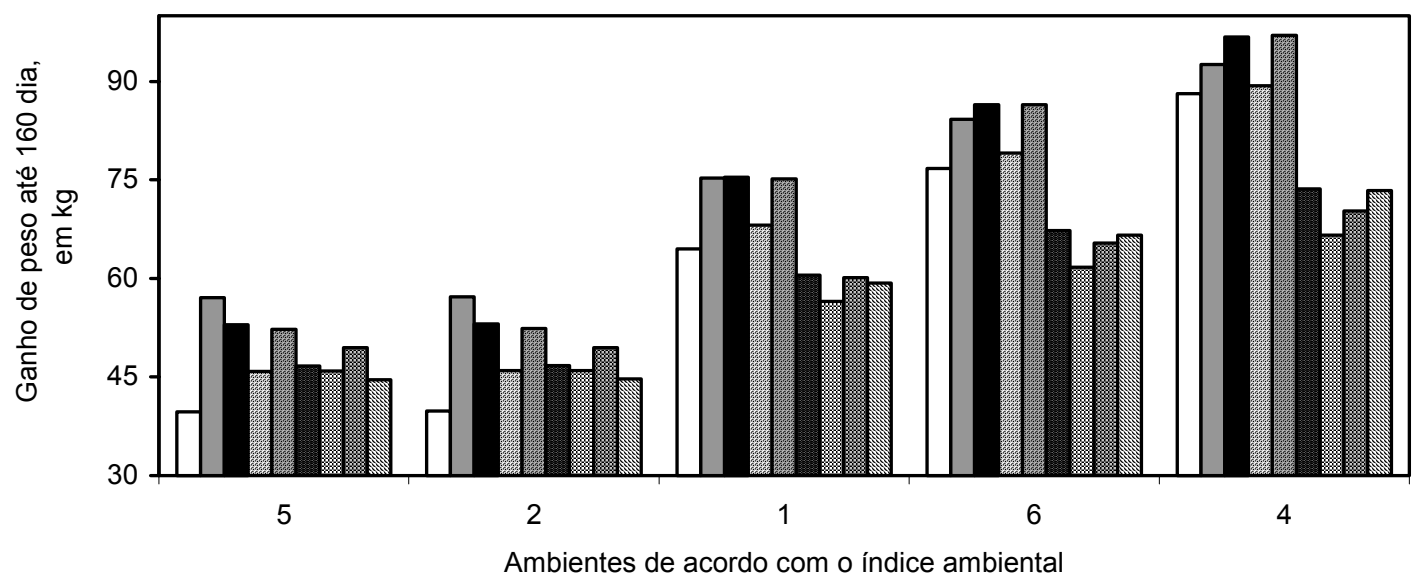

Figura 3. Comportamento da composição genotípica (CG-i) de acordo com cada índice ambiental, para a característica ganho de peso até 160 dias pós-desmama (GP160)

Os resultados deste estudo são consistentes com os de Rezende e Ferreira (2001), no tocante à eficiência da técnica proposta por Eberhart e Russel (1966), em discriminar combinações genotípicas frente aos diferentes ambientes criatórios. Todavia, Rezende e Ferreira (2001) classificaram os genótipos avaliados de maneira diferente, em virtude de utilizarem o critério proposto pela metodologia preconizada por Toler (1990) e não a classificação citada acima.

\section{CONCLUSÕES}

As combinações genotípicas dependem, em grande parte, do ambiente avaliado, sugerindo que as recomendações das melhores combinações genotípicas sejam específicas para cada ambiente avaliado. Nesses casos, a análise de adaptabilidade pelo método da regressão pode ser útil em discriminar o comportamento das combinações genotípicas dentro dos diferentes ambientes. Caso seja necessário avaliar o comportamento dos componentes de variância atribuídos aos desvios da regressão das combinações genotípicas, recomenda-se a utilização do coeficiente de determinação, para auxiliar nas tomadas de decisões.

\section{REFERÊNCIAS BIBLIOGRÁFICAS}

BALIEIRO, J.C.C. Heterogeneidade de variância na avaliação genética de bovinos da raça Nelore. 2001. 88 f. Tese (Doutorado em Genética e Melhoramento) - Universidade Federal de Viçosa, Viçosa, MG.

BROWN, M.A.; BROWN, A.H.; JACKSON, W.G. et al. Genotype $x$ environment interactions in postweaning performance to yearling in Angus, Brahman, and reciprocal-cross calves. J. Anim. Sci., v. 71, p.3273-3279, 1993.

BURROW, H.M. Variances and covariances between productive and adaptive traits and temperament in a composite breed of tropical beef cattle. Liv. Prod. Sci., v. 70, p. 213-233, 2001. 
CRUZ, C.D. Programa Genes: aplicativo computacional em genética e estatística. Viçosa, MG: UFV, 2001. 648p.

CRUZ, C.D.; REGAZZI, A.J. Modelos biométricos aplicados ao melhoramento genético. Viçosa: UFV, 1994. 390p.

CRUZ, C.D.; REGAZZI, A.J. Modelos biométricos aplicados ao melhoramento genético. 2.ed./rev.Viçosa: UFV, 2001. 390 p.

CRUZ, C.D.; TORRES, R.A.; VENCOVSKY, R. An alternative approach to the stability analysis proposed by Silva e Barreto. Rev. Bras. Genet., v.12, p.567-580, 1989.

EBERHART, S.A.; RUSSEL, W.A. Stability parameters for comparing varieties. Crop Sci., v.6, p.36-40, 1966.

FERREIRA, V.C.P.; PENNA, V.M.; BERGMANN, J.A.G. et al. Interação genótipoambiente em algumas características produtivas de gado de corte no Brasil. Arq. Bras. Med. Vet. Zootec., v.53, p. 385-392, 2001.

GAUCH JUNIOR, H.G.; ZOBEL, R.W. Predictive and postdictive success of statistical analysis of yield trials. Theor. Appl. Genet., v.76, p.1-10, 1988.

LEE, D.H.; BERTRAND, J.K. Investigation of genotype $\mathrm{x}$ country interactions for growth traits in beef cattle. J. Anim. Sci., v. 80, p.330-337, 2002.

LINS, C.S.; BINNS, M.R. A superiority measure of cultivar performance for cultivar x location data. Can. J. Plant. Sci., v.68, p.193-198, 1988.

MATTOS, D.; BERTRAND, J.K.; MISZTAL, I. Investigation of genotype $\mathrm{x}$ environment interactions for weaning weight for Herefords in three countries. J. Anim. Sci., v.78, p.2121-2126, 2000 .

MEYER, K. Estimates of variances due to sire $\mathrm{x}$ herd interactions and environmental covariances between paternal half-sibs for first lactation dairy production. Liv. Prod. Sci., v.17, p.95-115, 1987.

NEWMAN, S.; REVERTER, A.; JOHNSTON, D.J. Pubered-crossbred perfomance and genetic evaluation of postweaning growth and carcass traits in Bos indicus x Bos Taurus crosses in Australia. J. Anim. Sci., v.80, p.1801-1908, 2002.

NOTTER, D.R.; TIER, B.; MEYER, K. Sire $x$ herd interaction for weaning weight in beef cattle. $J$. Anim. Sci., v.70, p.2359-2365, 1992.

OJANGO, J.M.K.; POLLOTT, G.E. The relationship between Hostein bull breeding values for milk yield derived in both the UK and Kenya. Liv. Prod. Sci., v.74, p.1-12, 2002.

RAMOS, A.A.; VALENCIA, E.F.T.; WECHSLER, F.S. Heterogeneidade de variâncias das características de produção de bovinos da raça Holandesa no trópico: I. Estratificação por nível de produção de rebanhos. In: REUNIÃO ANUAL DA SOCIEDADE BRASILEIRA DE ZOOTECNIA, 33., 1996. Fortaleza. Anais... Fortaleza: SBZ, 1996. p.71-73.

REZENDE, D.M.L.C.; FERREIRA, D.F. Comparação de métodos de estimação da estabilidade fenotípica em animais da raça Holandesa. Pesq. Agropec. Bras., v.36, p.179-186, 2001.

REZENDE, D.M.L.C.; FERREIRA, D.F.; GONÇALVES, T.M. Avaliação da estabilidade fenotípica para produção de leite em animais da raça Holandesa. Rev. Bras. Zootec., v.28, p.735742, 1999.

RORATO, P.; LÔBO, R.B.; MARTINS FILHO, R. et al. Efeito da interação genótipo-ambiente sobre a produção de leite da raça Holandesa, no Estado do Paraná. Rev. Bras. Zootec., v.23, p.859-869, 1994.

RORATO, P.N.; MARTINEZ, M.L.; VERNEQUE, R.S. et al. Estudo do efeito da interação genótipoambiente sobre a produção de leite da raça Holandesa no Brasil. In: REUNIÃO ANUAL DA SOCIEDADE BRASILEIRA DE ZOOTECNIA, 33., 1996, Fortaleza. Anais... Fortaleza: SBZ, 1996. p.1-2.

TESS, M.W.; KRESS D.D.; BURFENING, P.J. et al. Sire $\mathrm{x}$ environment interactions in Simentalsired calves. J. Anim. Sci., v. 49, p. 964-971, 1979.

TOLER, J.E. Patterns of genotypic performance over environmental arrays. 1990. 154 f. Thesis (PhD) - Clemson University, Clemson, SC.

TORRES, R.A.; BERGMANN, J.A.G.; COSTA, C.N. Heterogeneidade de variância na avaliação genética de bovinos da raça Holandesa no Brasil. Rev. Bras. Zootec., v.29, p.1050-1059, 2000.

USER'S guide: statistic. Version 6.12. Cary, NC: SAS Institute, 1985. 168p.

ZWALD, N.R.; WEIGEL, K.A.; FIKSE, W.F. et al. Identification of factors that cause genotype by environment interaction between herds of Hostein cattle in seventeen Countries. J. Dairy Sci., v.86, p.1009-1018, 2003. 